\title{
Graphic requirements for multistationarity
}

\author{
Christophe Soulé \\ CNRS et Institut des Hautes Études Scientifiques
}

2-06-2003

IHÉS 35 Route de Chartres 91440 Bures-sur-Yvette France Tel:33160926600

Fax: 33160926609

Email: soule@ihes.fr 


\begin{abstract}
We discuss properties which must be satisfied by a genetic network in order for it to allow differentiation.

These conditions are expressed as follows in mathematical terms. Let $F$ be a differentiable mapping from a finite dimensional real vector space to itself. The signs of the entries of the Jacobian matrix of $F$ at a given point $a$ define an interaction graph, i.e. a finite oriented finite graph $G(a)$ where each edge is equipped with a sign. René Thomas conjectured twenty years ago that, if $F$ has at least two non degenerate zeroes, there exists $a$ such that $G(a)$ contains a positive circuit. Different authors proved this in special cases, and we give here a general proof of the conjecture. In particular, we get this way a necessary condition for genetic networks to lead to multistationarity, and therefore to differentiation.

We use for our proof the mathematical literature on global univalence, and we show how to derive from it several variants of Thomas' rule, some of which had been anticipated by Kaufman and Thomas.
\end{abstract}


When studying interactions in a system of biochemical compounds, it is quite rare that one obtains quantitative results. One can show that, in a given tissue, (the product of) a gene $A$ is an activator (or a repressor) of the expression of a gene $B$, but the strength of this interaction, the concentrations and their kinetics are usually unknown. The resulting information is essentially summarized by an interaction graph $G$, by which one means a finite oriented graph together with a sign for every edge. The vertices correspond to the members of the network, and there is a positive (resp. negative) edge from $A$ to $B$ when $A$ activates (resp. represses) the synthesis of $B$. Note that there can exist both a positive and a negative edge from $A$ to $B$, since $A$ can activate $B$ at some concentration and repress it at some other.

These interaction graphs can be quite complicated. It is therefore very desirable to find ways to use them to restrict the possible behaviour of the network they represent. In this paper, we shall address the question of when the network is susceptible to have several stationary states. A beautiful conjecture of R. Thomas [13] asserts that a necessary condition for multistationarity is that $G$ has an (oriented) circuit $C$ which is positive, i.e. such that the product of the signs of the edges of $C$ is positive, at least in part of phase space. This was already proved in several cases [11] [12] [7] [3], and we shall present a proof in the general case (Theorem 1).

To formulate the question in mathematical terms, we choose a continuous model. Let $n \geq 1$ be an integer, and let $F$ be a differentiable map from $\mathbb{R}^{n}$ to itself. The evolution of a network of $n$ compounds can be modelled by the differential system

$$
\frac{d x}{d t}=F(x),
$$

where $x$ is a differential path in $\mathbb{R}^{n}$ : the components of $x(t)$ are the different concentrations at time $t$. To ask whether this system has several stationary states amounts to ask whether $F$ has several zeroes. Given any $a \in \mathbb{R}^{n}$, the interaction graph $G(a)$ is defined from the signs of the partial derivatives $\left(\partial f_{i} / \partial x_{j}\right)(a)$ of the components of $F=\left(f_{i}\right)$ at a (see 2.1 below for a precise definition) : a positive (resp. negative) sign indicates that $j$ is an activator (resp. a repressor) of $i$. The precise formulation of Thomas' rule is then the following: if $F$ has at least two (non-degenerate) zeroes in $\mathbb{R}^{n}$, there exists $a \in \mathbb{R}^{n}$ such that $G(a)$ contains a positive circuit.

The main remark leading to a proof of this assertion is the following. If $F$ has several zeroes, it cannot be univalent (i.e. one to one). Therefore, if we know sufficient conditions for $F$ to be univalent, their negation will give necessary conditions for $F$ to have several zeroes. And we might then deduce properties of $G(a)$ from these necessary conditions.

It turns out that finding sufficient conditions for $F$ to be univalent is a classical issue in mathematical economy, when one wants to know that the factor prices are uniquely determined by the prices for goods. Several results 
have been obtained with this application in mind. G. Gale and H. Nikaido [5] gave an elegant criterion of univalence in terms of the Jacobian matrix $J(x)=$ $\left(\partial f_{i} / \partial x_{j}\right)(x)$ : assume that, for every $x \in \mathbb{R}^{n}$, the principal minors (resp. the determinant) of $J(x)$ are nonnegative (resp. is positive); then $F$ is univalent. As we shall see, this result leads precisely to a proof of Thomas' conjecture (Theorem 1).

Following the same line of arguments, we can also apply variants of the theorem of Gale-Nikaido, which are discussed for instance in the book of T. Parthasarathy [10]. A motivation for doing so is that one may want to restrict the domain of $F$ to be the closed positive quadrant (since a concentration cannot be negative). In general, $F$ will be defined on a product $\Omega$ of $n$ intervals, open or not. However, in this generality, there exist counter-examples to the condition of Thomas (see 3.5). But, when $F$ has at least two zeroes, we can still find properties of its interaction graphs (Theorem 5), as a consequence of a univalence theorem of Gale-Nikaido ([5] , [10]). Furthermore, it is often the case that, when defining the interaction graph of several biochemical compounds, the degradation of each of them is not taken into account. We show in Theorem 6 that this makes Thomas' rule valid in full generality.

Another refinement of the theorem of Gale-Nikaido, due to C.B. Garcia and W.I. Zangwill [6], applies to the case where $\Omega$ is closed and bounded. This leads to Theorem 7 which, in turn, for general $\Omega$, gives some information on the location of the zeroes of $F$ (Theorem 8 ).

The economist P.A. Samuelson imagined a stronger univalence criterion that those above. It is not true for general $F$, but L.A. Campbell proved it when $\Omega=\mathbb{R}^{n}$ and $F$ is algebraic, e.g. when each component of $F$ is the quotient of two real polynomials. In Theorem 9 we translate his result in terms of properties of the interaction graph.

One can expect more graphical requirements for multistationarity. One of them is a conjecture of M. Kaufman and R. Thomas [15], which is stronger than the original Thomas' conjecture. We have been unable to prove this assertion, but we obtained some evidence for it in Theorems 3,4 and 5 .

The paper is organized as follows. The first section gives definitions about graphs, matrices and determinants. It shows basic lemmas, which are standard knowledge in the literature on interaction graphs. In section two we define the interaction graphs $G(a)$ and we state the conjectures of Thomas and KaufmanThomas. In the third paragraph we prove the conjecture of Thomas and we give some (counter)examples. In section four we discuss the conjecture of KaufmanThomas. Next, we give results when the domain $\Omega$ is not necessarily open, and we refine them in section six. Finally, we discuss the case of an algebraic map in the last section.

The literature on the question studied here is scattered in several different journals, and it has been crucial for me to receive papers from different authors. 
I want to thank for that J. Aracena, O. Cinquin, J. Demongeot, J.L. Gouzé, M.S. Gowda, M. Kaufman, D. Thiéfry and R. Thomas. I am also grateful to the participants of the "Séminaire d'initiation à la génomique fonctionnelle" of IHÉS, and especially to F. Képès, who explained the importance of genetic networks, and first mentioned to me the rule of Thomas. Finally, I am extremely thankful to M. Kaufman and R. Thomas for several discussions, where they patiently and carefully explained their ideas, provided examples and encouraged me to do this work.

\section{Graphs and matrices}

\section{1}

An interaction graph $G=(V, E, \operatorname{sgn})$ is a finite oriented graph $(V, E)$ together with a sign map sgn : $E \rightarrow\{ \pm 1\}$. In other words, $V$ (the vertices) and $E$ (the edges) are two finite sets and each edge $e \in E$ has an origin $o(e) \in V$ and an endpoint $t(e) \in V$ (it may happen that $o(e)=t(e)$ ).

A circuit in $G$ is a sequence of edges $e_{1}, \ldots, e_{k}$ such that $o\left(e_{i+1}\right)=t\left(e_{i}\right)$ for all $i=1, \ldots, k-1$ and $t\left(e_{k}\right)=o\left(e_{1}\right)$.

A hooping is a collection $C=\left\{C_{1}, \ldots, C_{k}\right\}$ of circuits such that, for all $i \neq j$, $C_{i}$ and $C_{j}$ do not have a common vertex. A circuit is thus a special case of hooping. We let $V(C)=\coprod_{i=1}^{k} V\left(C_{i}\right)$ be the (unordered) set of vertices of $C$. This set $V(C)$ will also be called the support of $C$. A hooping is called Hamiltonian when its set of vertices is maximal, i.e. $V(C)=V$. Note that hoopings are called "generalized circuits", or "g-circuits" in [4], and Hamiltonian ones are called "full circuits" in [15].

The sign of a circuit $C$ is

$$
\operatorname{sgn}(C)=\prod_{e \in C} \operatorname{sgn}(e) \in\{ \pm 1\}
$$

When $\operatorname{sgn}(C)=+1$ (resp. -1 ) we say that $C$ is positive (resp. negative). The sign of a hooping $C$ is

$$
\operatorname{sgn}(C)=(-1)^{p+1},
$$

where $p$ is the number of positive circuits in $C$ (cf. [4]).

Given any subset $I \subset V$ we let $\tau_{I} G$ the interaction graph obtained from $G$ by changing the sign of every edge $e \in E$ such that $t(e) \in I$. Given any permutation $\sigma \in \operatorname{Aut}(V)$ of the vertices, we let $\sigma G$ be the interaction graph obtained from $G$ by replacing each edge $j \rightarrow i$ by an edge $j \rightarrow \sigma(i)$, with the same sign. 


\section{2}

Let $n \geq 1$ be an integer and $A=\left(a_{i j}\right)$ an $n$ by $n$ real matrix. We can attach to $A$ an interaction graph $G$ as follows. The set of vertices of $G$ is $\{1, \ldots, n\}$. There is an edge $e$ with $o(e)=j$ and $t(e)=i$ if and only if $a_{i j} \neq 0$. The sign of $e$ is the sign of $a_{i j}$.

Given any subset $I \subset\{1, \ldots, n\}$, the principal minor of $A$ with support $I$ is the real number $\operatorname{det}\left(A_{I}\right)$, where $A_{I}$ is the square matrix $\left(a_{i j}\right)_{i, j \in I}$. By definition

$$
\operatorname{det}\left(A_{I}\right)=\sum_{\sigma \in \Sigma_{I}} \varepsilon(\sigma) \prod_{i \in I} a_{i \sigma(i)},
$$

where $\Sigma_{I}$ is the group of permutations of $I$ and $\varepsilon(\sigma)$ is the signature of $\sigma$ (Recall that $\varepsilon$ is defined by the equalities $\varepsilon\left(\sigma \sigma^{\prime}\right)=\varepsilon(\sigma) \varepsilon\left(\sigma^{\prime}\right)$ for all $\sigma, \sigma^{\prime} \in \Sigma_{I}$ and $\varepsilon(\sigma)=-1$ when $\sigma \in \Sigma_{I}$ is a transposition).

For any $\sigma \in \Sigma_{I}$ we let

$$
a(\sigma)=\varepsilon(\sigma) \prod_{i \in I} a_{i \sigma(i)}
$$

so that

$$
\operatorname{det}\left(A_{I}\right)=\sum_{\sigma \in \Sigma_{I}} a(\sigma) .
$$

When $a(\sigma) \neq 0$, we let $\operatorname{sgn}(a(\sigma))= \pm 1$ be its sign.

Let $D=\operatorname{diag}\left(d_{1}, \ldots, d_{n}\right)$ be a diagonal $n$ by $n$ real matrix and $I \subset \Sigma$ any subset. It follows from the definition (2) that

$$
\operatorname{det}\left((A+D)_{I}\right)=\sum_{J \subset I} \operatorname{det}\left(A_{J}\right) \prod_{i \in I-J} d_{i} .
$$

Given $I \subset V$ any subset, we let $\tau_{I} A$ be the matrix obtained from $A$ by replacing $a_{i j}$ by $-a_{i j}$ whenever $i \in I$. Given any $\sigma \in \Sigma_{n}=\operatorname{Aut}(V)$ we let $\sigma A$ be the product of $A$ with the permutation matrix defined by $\sigma$. Clearly

$$
G\left(\tau_{I} A\right)=\tau_{I} G(A)
$$

and

$$
G(\sigma A)=\sigma G(A) .
$$

\section{3}

We keep the notation of the preceeding paragraph. Note that, given any permutation $\sigma \in \Sigma_{I}$, there is a unique decomposition

$$
I=I_{1} \amalg I_{2} \amalg \ldots \amalg I_{k}
$$


of $I$ into a disjoint union of nonempty subsets such that the restriction $\sigma_{\alpha}$ of $\sigma$ to $I_{\alpha}$ is a cyclic permutation for all $\alpha=1, \ldots, k$. Let $C\left(\sigma_{\alpha}\right)$ be the circuit of $G$ with edges $\left(i, \sigma_{\alpha}(i)\right), i \in I_{\alpha}$ (note that $a_{i \sigma_{\alpha}(i)} \neq 0$ since $a(\sigma) \neq 0$ ). We denote by $C(\sigma)$ the hooping of $G$ which is the disjoint union of the circuits $C\left(\sigma_{\alpha}\right)$, $\alpha=1, \ldots, k$.

When $X$ is a finite set, we let \# $(X)$ be its cardinality. The following lemma is due to J.Eisenfeld and C.DeLisi ([4], Appendix, Lemma 2), and is probably at the origin of the definition (1).

Lemma 1. Let $I \subset\{1, \ldots, n\}$ be any subset and let $\sigma \in \Sigma_{I}$ be such that $a(\sigma) \neq 0$. Then the following identity holds

$$
\operatorname{sgn}(C(\sigma))=\operatorname{sgn}(a(\sigma))(-1)^{\#(I)+1} .
$$

Proof. Since $\varepsilon(\sigma)=\sum_{\alpha=1}^{k} \varepsilon\left(\sigma_{\alpha}\right)$ and $I=\coprod_{\alpha} I_{\alpha}$, we get from (3) that

$$
a(\sigma)=\prod_{\alpha} a\left(\sigma_{\alpha}\right) .
$$

For each $\alpha=1, \ldots, k$, since $\sigma_{\alpha}$ is cyclic we have

$$
\varepsilon\left(\sigma_{\alpha}\right)=(-1)^{\#\left(I_{\alpha}\right)+1} .
$$

The circuit $C\left(\sigma_{\alpha}\right)$ is positive if and only if $\prod_{i \in I_{\alpha}} a_{i \sigma_{\alpha}(i)}$ is positive, in which case we get from (3) that

$$
\operatorname{sgn}\left(a\left(\sigma_{\alpha}\right)\right)=(-1)^{\#\left(I_{\alpha}\right)+1} .
$$

When $C\left(\sigma_{\alpha}\right)$ is negative we get

$$
\operatorname{sgn}\left(a\left(\sigma_{\alpha}\right)\right)=(-1)^{\#\left(I_{\alpha}\right)} .
$$

Therefore, by (5), (6), (7), we get

$$
\operatorname{sgn}(a(\sigma))=(-1)^{p}(-1)^{\sum_{\alpha} \#\left(I_{\alpha}\right)}=(-1)^{p}(-1)^{\#(I)},
$$

where $p$ is the number of positive circuits in $C(\sigma)$. Since, by $(1), \operatorname{sgn}(C(\sigma))=$ $(-1)^{p+1}$, the lemma follows.

\section{4}

From Lemma 1 we get the following:

Lemma 2. Let $A$ be an $n$ by $n$ real matrix and let $I \subset\{1, \ldots, n\}$ be any subset. 
i) Assume that $\operatorname{det}\left(-A_{I}\right)$ is negative (resp. positive). Then there exists a positive (resp. negative) hooping in $G(A)$ with support $I$.

ii) Assume $\operatorname{det}\left(-A_{I}\right)=0$. Then either there exist two hoopings in $G(A)$ with opposite signs and support I, or there is no hooping in $G(A)$ with support I.

Proof. From (2) we get

$$
\operatorname{det}\left(-A_{I}\right)=(-1)^{\#(I)} \operatorname{det}\left(A_{I}\right) .
$$

Therefore, by Lemma 1,

$$
\operatorname{det}\left(-A_{I}\right)=-\sum_{\sigma \in \Sigma_{I}} \operatorname{sgn}(C(\sigma))|a(\sigma)| .
$$

Assume $\operatorname{det}\left(-A_{I}\right)$ is negative (resp. positive). Then, by (8), there exists $\sigma \in \Sigma_{I}$ such that $C(\sigma)$ is positive (resp. negative). This proves i).

If $\operatorname{det}\left(-A_{I}\right)=0$, either there exist two summands with opposite signs on the right hand side of (8), or $a(\sigma)$ is zero for all $\sigma \in \Sigma_{I}$. This proves ii), since every hooping of $G(A)$ with support $I$ is of the form $C(\sigma)$ for some $\sigma \in \Sigma_{I}$.

\section{Conjectures on multistability}

\section{1}

Let $\Omega_{i}$ be a nonempty interval in $\mathbb{R}$ :

$$
\left.\Omega_{i}=\right] a_{i}, b_{i}\left[,\left[a_{i}, b_{i}[,] a_{i}, b_{i}\right] \text { or }\left[a_{i}, b_{i}\right]\right.
$$

with $a_{i} \geq-\infty$ and $b_{i} \leq+\infty$. Denote by $\Omega \subset \mathbb{R}^{n}$ the product $\Omega=\prod_{i=1}^{n} \Omega_{i}$. Consider a map

$$
F: \Omega \rightarrow \mathbb{R}^{n}
$$

which is differentiable, i.e. such that, for each $i, j \in\{1, \ldots, n\}$ and any $a \in \Omega$, the $i$-th component $f_{i}$ of $F$ has a partial derivative $\frac{\partial f_{i}}{\partial x_{j}}(a)$ at the point $a$ and

$$
f_{i}(x)=f_{i}(a)+\sum_{j=1}^{n} \frac{\partial f_{i}}{\partial x_{j}}(a)\left(x_{j}-a_{j}\right)+o(\|x-a\|),
$$

where $\|x-a\|$ is the norm of $x-a$ and $o$ is the Landau $o$-symbol (we could also use a weaker notion of differentiability, see [8]). The map $F$ is called $C^{1}$ when every partial derivative $\partial f_{i} / \partial x_{j}$ is continuous on $\Omega$.

For any $a \in \Omega$, the Jacobian of $F$ at $a$ is the $n$ by $n$ real matrix

$$
J(a)=J(F)(a)=\left(\frac{\partial f_{i}}{\partial x_{j}}(a)\right) .
$$


For any $a \in \Omega$ we let

$$
G(a)=G(J(a))
$$

be the interaction graph of $J(a)$, defined as in 1.2. We also let $G(F)$ be the interaction graph defined as follows. Its set of vertices is $V=\{1, \ldots, n\}$. Given $i$ and $j$ in $V$, there is at most one positive (resp. negative) edge from $j$ to $i$; it exists if and only if there is a positive (resp. negative) path in $G(a)$ for some $a \in \Omega$. In other words, $G(F)$ is the "superposition" of all the interaction graphs $G(a)$.

Given any subset $I \subset V$, we let $\tau_{I} F$ be the map obtained from $F=\left(f_{i}\right)$ by replacing $f_{i}$ by $-f_{i}$ when $i \in I$. Given $\sigma \in \Sigma_{n}$, we let $\sigma F=\left(f_{\sigma^{-1}(i)}\right)$. The interaction graphs of $\sigma \tau_{I} F$ are $\sigma \tau_{I} G(a), a \in \Omega$, and $\sigma \tau_{I} G(F)$.

\section{2}

We shall be interested in the set of zeroes of $F$, i.e. the points $a \in \Omega$ such that $F(a)=0$. They can be viewed as the stationary states of the system of differential equations

$$
\frac{d x(t)}{d t}=F(x),
$$

where $x(t)$ is a differentiable mapping from a real interval to $\Omega$.

We say that a zero $a$ of $F$ is nondegenerate when $\operatorname{det}(J(a)) \neq 0$.

\subsection{Conjecture 1. (Thomas [13])}

Assume that $\Omega$ is open and that $F$ has at least two nondegenerate zeroes in $\Omega$. Then there exists $a \in \Omega$ such that $G(a)$ contains a positive circuit.

Remark. This conjecture has already been proved in several cases. First, it is known to hold when the signs of the entries in $J(a)$ are independent of $a \in \Omega$ [11] [7]; see also [12] and the remark in 5.2 below. It was also shown in [3] for stable stationary states when $\Omega$ contains the positive quadrant and $f_{i}(x)>0$ whenever $x_{i}=0$.

\subsection{Conjecture 2. (Kaufman-Thomas [15])}

Under the same assumption as Conjecture 1,

i) Either there exist $a \in \Omega$ such that $G(a)$ has a positive Hamiltonian hooping and a negative Hamiltonian hooping.

ii) Or there is a cyclic permutation $\sigma$ of a subset of $\{1, \ldots, n\}$ and there exist $a, b \in \Omega$ such that the circuit $C(\sigma)$ in $G(a)$ (resp. in $G(b)$ ) is positive (resp. negative).

Note that Conjecture 2 for $F$ implies Conjecture 1 for $F$. It is quite different though, since positive and negative circuits play in it a symmetric role. In case 
i) we shall say that " $G$ has two Hamiltonian hoopings of opposite signs" and in case ii) we shall say that " $G$ has an ambiguity". In the latter case, $G(F)$ contains two circuits with the same ordered set of vertices and opposite signs (but ii) is stronger than that statement).

\section{A proof of Thomas' Conjecture 1}

\section{1}

Theorem 1. Assume $\Omega$ is open and $F$ has at least two nondegenerate zeroes in $\Omega$. Then, for every $I \subset V$ and every $\sigma \in \Sigma_{n}$, there exists $a \in \Omega$ such that $\sigma \tau_{I} G(a)$ has a positive circuit. In particular, Thomas' Conjecture 1 holds true.

Since $\sigma \tau_{I} G(x)=G\left(\sigma \tau_{I} J(x)\right)$ and since $\sigma \tau_{I}(F)$ satisfies the hypotheses of Theorem 1 if and only if $-F$ does, we just have to check that, for some $a \in \Omega$, the interaction graph $G(-J(a))$ has a positive circuit. According to Lemma 2 i), it will be enough to show that, for some $a \in \Omega$, a principal minor of $-J(a)$ is negative. In other words:

Theorem 1'. Assume that, for every $a \in \Omega$, all the principal minors of $-J(a)$ are nonnegative. Then $-F$ can have at most one nondegenerate zero.

\section{2}

For any positive real number $\lambda$ let

$$
\phi_{\lambda}(x)=-F(x)+\lambda x .
$$

Since

$$
J\left(\phi_{\lambda}\right)=-J(F)+\operatorname{diag}(\lambda, \ldots, \lambda),
$$

it follows from (4) that, under the hypotheses of Theorem 1', each principal minor of $J\left(\phi_{\lambda}\right)$ is positive on $\Omega$. According to Gale and Nikaido, [5] Theorem 4 , this implies that $\phi_{\lambda}$ is univalent. The following proposition ends the proof of Theorem 1':

Proposition 1. Let $\phi=\Omega \rightarrow \mathbb{R}^{n}$ be a differentiable map defined on an open set $\Omega \subset \mathbb{R}^{n}$. Assume that, for all $\lambda>0$, the map

$$
\phi_{\lambda}(x)=\phi(x)+\lambda x
$$

is univalent. Then $\phi$ can have at most one nondegenerate zero in $\Omega$. 


\section{3}

The proof of Proposition 1 proceeds as [5] Theorem 4' and [10], IV, Theorem 4 , p. 35. Assume $a$ and $b$ are two nondegenerate zeroes of $\phi$ in $\Omega$. According to [1], XII, $\S 2.9$, p. 477, we can choose open neighborhoods $U_{a}$ and $U_{b}$ of $a$ and $b$ respectively such that $\bar{U}_{a} \cap \bar{U}_{b}=\emptyset, \bar{U}_{a} \subset \Omega, \bar{U}_{b} \subset \Omega, a$ (resp. $b$ ) is the unique zero of $\phi$ in $\bar{U}_{a}$ (resp. $\left.\bar{U}_{b}\right)$, and the degrees $\operatorname{deg}\left(\phi, \bar{U}_{a}, a\right)$ and $\operatorname{deg}\left(\phi, \bar{U}_{b}, 0\right)$ are equal to \pm 1 . Arguing as in [10] (or [5]), loc.cit., we get

$$
1=\operatorname{deg}\left(\phi, \bar{U}_{a} \cup \bar{U}_{b}, 0\right)=\operatorname{deg}\left(\phi, \bar{U}_{a}, 0\right)+\operatorname{deg}\left(\phi, \bar{U}_{b}, 0\right),
$$

hence a contradiction.

q.e.d.

\section{4}

The restriction to nondegenerate zeroes of $F$ in Theorem 1 is necessary. For example, if $n=2, \Omega=\mathbb{R}^{2}$ and

$$
F(x, y)=\left(-x y^{2},-y\right)
$$

we have

$$
-J F(x, y)=\left(\begin{array}{cc}
y^{2} & 2 x y \\
0 & 1
\end{array}\right) .
$$

Clearly $G$ has no positive circuit. However $F(x, 0)=0$ for any $x \in \mathbb{R}$.

\section{5}

It is also essential that $\Omega$ be open. Let $\Omega$ be the set of $(x, y) \in \mathbb{R}^{2}$ such that $x \geq 0$ and $y \geq 0$. Consider the map $F: \Omega \rightarrow \mathbb{R}^{2}$ defined by

$$
F(x, y)=\left((y-2)^{2}-x^{2}-1,4 x-4 x y\right) .
$$

We get

$$
-J F(x, y)=\left(\begin{array}{cc}
2 x & 4-2 y \\
2 y-4 & 2 x
\end{array}\right) .
$$

Therefore, by (8), $G(F)$ does not have any positive circuit. On the other hand both $(0,3)$ and $(0,1)$ are nondegenerate zeroes of $F$ in $\Omega$. We shall discuss the case of an arbitrary $\Omega$ in sections five and six below.

\section{On the conjecture of Kaufman-Thomas}

\section{1}

We first assume that $n=2$. Let $\Omega \subset \mathbb{R}^{2}$ be as in 2.1 and open, with coordinates $x$ and $y$. For any $h: \Omega \rightarrow \mathbb{R}$ we write $h \equiv 0$ to mean that $h(a)=0$ for every 
$a \in \Omega$. If $h$ is differentiable, we let $h_{x}$ (resp. $h_{y}$ ) be its partial derivative with respect to the first (resp. second) variable.

Let

$$
F=(f, g): \Omega \rightarrow \mathbb{R}^{2}
$$

be a differentiable mapping.

Theorem 2. Assume that $F$ has at least two nondegenerate zeroes in $\Omega$. Then, one of the following conditions holds:

i) G has two Hamiltonian hoopings of opposite signs;

ii) G has an ambiguity;

iii) $f_{x} g_{y} \equiv 0$ but $f_{x} \not \equiv 0$ and $g_{y} \not \equiv 0$.

Proof. Assume that $G$ has no ambiguity, and that its Hamiltonian hoopings have all the same sign. If, in addition, $f_{x} g_{y} \not \equiv 0$, since the sign of $f_{x}(a)$ (resp. $g_{y}(a)$ ) is independent of $a$, we can multiply $f$ and $g$ by \pm 1 to get to the case where $f_{x}(a) \geq 0$ and $g_{y}(a) \geq 0$ for all $a \in \Omega$. It follows that $f_{x}(a) g_{y}(a) \geq 0$ and, since all Hamiltonian hoopings have the same sign, $f_{y}(a) g_{x}(a) \leq 0$ (by Lemma 1). Therefore Theorem 1' applies to $F$ and we conclude that $F$ has at most one nondegenerate zero.

Assume now that $g_{y} \equiv 0$. After multiplying $f$ and $g$ by \pm 1 we can assume that $f_{x} \geq 0$ and $f_{y} g_{x} \leq 0$. Once again, Theorem 1 'implies that $F$ has at most one nondegerate zero. q.e.d.

\section{2}

With an additional assumption, Conjecture 2 is true for all $n \geq 2$ :

Theorem 3. Let $F: \Omega \rightarrow \mathbb{R}^{n}$ be a differentiable mapping such that $\Omega$ is open and $F$ has at least two nondegenerate zeroes. Then, one of the following conditions holds:

i) G has two Hamiltonian hoopings with opposite signs;

ii) G has an ambiguity;

iii) for any point $a \in \Omega$ there exists $i \in\{1, \ldots, n\}$ such that $G(a)$ does not contain an edge from $i$ to itself.

Proof. Assume that all Hamiltonian hoopings in $G$ have the same sign, that $G$ has no ambiguity and that, for some $a \in \Omega$, and for any $i \in\{1, \ldots, n\}$, there is an edge in $G(a)$ from $i$ to itself. The last condition means that all diagonal entries in $J(a)$ are nonzero. After multiplying each component of $F$ by \pm 1 we can assume that all diagonal entries of $J(a)$ are positive. Let $C$ be any hooping 
in $J(a)$, and let $I$ be its set of vertices. The disjoint union of $C$ with all the circuits $i \rightarrow i, i \in\{1, \ldots, n\}-I$, is a Hamiltonian hooping of $G(a)$. Its sign must be the sign of the Hamiltonian hooping which is the disjoint union of all the positive circuits $i \rightarrow i, i \in\{1, \ldots, n\}$, namely $(-1)^{n+1}$. We conclude that

$$
\operatorname{sgn}(C)=(-1)^{\#(I)} \text {. }
$$

By Lemma 1, this implies that $C=C(\sigma)$ with $\operatorname{sgn}(a(\sigma))=+1, \sigma \in \Sigma_{I}$. Since $G$ has no ambiguity, for any $\sigma \in \Sigma_{I}$ we have $\operatorname{sgn}(a(\sigma)) \geq 0$ in $\Omega$. Therefore, for any $x \in \Omega$, all the principal minors of $J(x)$ are nonnegative. Applying Theorem 1', we conclude that $F$ has at most one nondegenerate zero.

\section{3}

Concerning the graph $G(F)$ (see 2.1) we can prove the following:

Theorem 4. Let $F: \Omega \rightarrow \mathbb{R}^{n}$ be a differentiable mapping such that $\Omega$ is open and $F$ has at least two nondegenerate zeroes. Then $G(F)$ has two Hamiltonian hoopings with opposite signs.

Proof. We first remark that we can find $a \in \Omega$ and $\sigma \in \Sigma_{n}$ such that none of the diagonal entries in $J(\sigma F)(a)$ is zero. Indeed these entries are, by definition, $\frac{\partial f_{\sigma^{-1}(j)}}{\partial x_{j}}(a), j=1, \ldots, n$, and, when $a$ is a non degenerate zero of $F$, it follows from (1) that, for some $\sigma \in \Sigma_{n}$,

$$
\prod_{i=1}^{n} \frac{\partial f_{i}}{\partial x_{\sigma(i)}}(a) \neq 0 .
$$

This proves the claim.

We may then choose $I$ such that all the diagonal entries in $J\left(\tau_{I} \sigma F\right)(a)$ are negative. In other words, for each $i \in\{1, \ldots, n\}$, the interaction graph $\tau_{I} \sigma G(a)$ contains a negative edge $i \rightarrow i$. On the other hand, by Theorem 1 , there exists $b \in \Omega$ such that $\tau_{I} \sigma G(b)$ has a positive circuit $C$. In $\tau_{I} \sigma G(F)$ the disjoint union of $C$ with the negative edges $i \rightarrow i, i \notin V(C)$, is a positive Hamiltonian hooping, when the union of all the negative edges $i \rightarrow i, i \in\{1, \ldots, n\}$, is a negative one.

It is thus enough to show that, if $\tau_{I} G(F)$ or $\sigma G(F)$ has two Hamiltonian hoopings with opposite signs, the same is true for $G(F)$. This is clear for $\tau_{I} G(F)$ and, for $\sigma G(F)$, it follows from Lemma 3 below.

\section{4}

Lemma 3. Let $G$ be any interaction graph, $\sigma \in \operatorname{Aut}(V)$ a permutation of its vertices, and $C$ an Hamiltonian hooping in $G$. The image of $C$ in the interaction graph $\sigma G$ is then an Hamiltonian hooping with sign $\varepsilon(\sigma) \operatorname{sgn}(G)$. 
Proof. Recall from 1.1 that $\sigma G$ is obtained from $G$ by replacing each edge $j \rightarrow i$ by an edge $j \rightarrow \sigma(i)$, with the same sign. As a collection of edges, $C$ has a well defined image $\sigma C$ in $\sigma G$. To check Lemma 3 we may assume that $\sigma$ is the transposition of two vertices $i$ and $j$ (transpositions span $\operatorname{Aut}(V)$ ).

Assume first that $i$ and $j$ are in the same circuit $C_{1}$ of $C$. Then all the circuits in $C$ other than $C_{1}$ are fixed by $\sigma$. The image of $C_{1}$ consists of two disjoints circuits $D_{1}$ and $D_{2}$. More precisely, if the vertices of $C_{1}$ are $12 \ldots i \ldots j \ldots m$ (as we can assume), we get

$$
\sigma C_{1}=D_{1} \coprod D_{2}
$$

where the sequence of vertices in $D_{1}$ (resp. $D_{2}$ ) is $12 \ldots i-1 j j+1 \ldots k 1$ (resp. $i i+1 \ldots j-1 i$ ). Furthermore, if $C_{1}$ has an even (resp. odd) number of negative edges, $D_{1}$ and $D_{2}$ will have the same (resp. a different) number of edges modulo two. From this it follows that $\sigma C$ is a Hamiltonian hooping such that, with the definition (1),

$$
\operatorname{sgn}(\sigma C)=-\operatorname{sgn}(C)
$$

as was to be shown.

Note that we also have

$$
C_{1}=\sigma D_{1} \coprod \sigma D_{2},
$$

therefore, by exchanging the roles of $C$ and $\sigma C$, the previous discussion applies also to the case where $i$ and $j$ lie in two different circuits of $C$.

q.e.d.

\section{The case of a domain which is not open}

\section{1}

We keep the notation of 2.1, where $\Omega$ is an arbitrary product of intervals and $F: \Omega \rightarrow \mathbb{R}^{n}$ is differentiable.

\section{Theorem 5.}

Assume that $F$ is not univalent. Then:

1) For every $\sigma \in \Sigma_{n}$,

i) Either there exists $a \in \Omega$ and $i \in V$ such that $\sigma G(a)$ does not contain any edge from $i$ to itself;

ii) Or, for any subset $I \subset V$, there exists $a \in \Omega$ such that $\tau_{I} \sigma G(a)$ has a positive circuit.

2) When the condition 1) i) above is not satisfied, $G(F)$ contains both a positive and a negative Hamiltonian hooping.

Proof. The map $F$ is univalent if and only if $\tau_{I} \sigma F$ is. So, to prove 1 ), we can restrict our attention to $-F$. Note also that i) is equivalent to the assertion 
that there exists $I \subset V$ such that $\sigma G(a)$ does not contain any hooping with support I. According to Lemma 2 ii), if none of the conclusions in 1) is true, all the principal minors of the Jacobian matrix of $-F$ are positive on $\Omega$. By the Gale-Nikaido theorem, [5] Theorem 4, this implies that $-F$ is univalent. This proves 1).

To prove 2), by replacing $F$ by $\tau_{I} F$ for an appropriate choice of $I$, we can assume that, for each vertex $i$ in $G(F)$, there is a negative edge from $i$ to itself. Since $G(F)$ also contains a positive circuit by 1 ), we get, as in 4.3 above, that $G(F)$ contains both a positive and a negative Hamiltonian hooping.

Remark.Since the condition 1) i) is often satisfied, when $\Omega$ is open Theorem 5 is much weaker than Theorems 1 and 4 .

\section{2}

Here is a variant of Theorem 5 .

Theorem 6. Assume given, for every $a \in \Omega$, a diagonal matrix $D(a)$ with positive entries. Assume $F$ is not univalent. Then, for some $a \in \Omega$, the interaction graph

$$
H(a)=G(J(a)+D(a))
$$

has a positive circuit.

Proof. Assume that, for any $a \in \Omega$, none of the circuits of $H(a)$ is positive. We know from Lemma 2 i) that all the principal minors of $-J(a)-D(a)$ are nonnegative. From (4) this implies that all the principal minors of $-J(a)$ are positive, and, again by [5] Theorem $4, F$ must be univalent.

Remark. Theorem 6 applies to the situation considered for instance in [14] and [9], where $-D(a)$ comes from "terms of decay", which are not taken into account when drawing the interaction graph. It was proven by Snoussi [12] when the signs of the entries of $J(a)$ are constant.

\section{On the location of stationary states}

\section{1}

Assume that $\Omega=\prod_{i=1}^{n}\left[a_{i}, b_{i}\right]$ is a closed bounded subset of $\mathbb{R}^{n}$. In that case, Garcia and Zangwill got a stronger result that Gale-Nikaido ([6], see also [10], $\mathrm{V}$, Theorem 1, p. 41). For any $I \subset V$ define $I^{c}=V-I$ and, for any $n$ by $n$ real matrix $A$, let

$$
m_{I}(A)=\operatorname{det}\left(A_{I^{c}}\right)
$$

and

$$
m_{i}(A)=m_{\{i\}}(A)
$$


for each $i \in V$. When $x$ lies in $\Omega$, we write $m_{I}(x)$ for $m_{I}(J(x))$ and $m_{i}(x)=$ $m_{i}(J(x))$. Define $I(x) \subset V$ as the set of vertices $i$ such that $x_{i}=a_{i}$ or $x_{i}=b_{i}$. The result of Garcia-Zangwill is the following

Theorem 7'. Assume $F$ is $C^{1}$ and that, for every $a \in \Omega$, and every subset $I \subset I(a)$,

$$
m_{I}(a) \prod_{i \in I} m_{i}(a)>0
$$

Then $F$ is univalent.

In particular, when $\left.a \in \stackrel{\circ}{\Omega}=\prod_{i=1}^{n}\right] a_{i}, b_{i}[, I(a)=\emptyset$ and the only assertion made is that $\operatorname{det} J(a)>0$.

When $F$ is only differentiable, see [8], p. 930, Remark.

\section{2}

Theorem 7' implies the following refinement of Theorem 5:

Theorem 7. Assume that $\Omega$ is bounded and closed, and that $F: \Omega \rightarrow \mathbb{R}^{n}$ is $C^{1}$. If $F$ is not univalent, for every $\sigma \in \Sigma_{n}$, one of the following conditions holds true:

i) There exists $a \in \Omega$ and $I \subset I(a)$ such that no hooping of $\sigma G(a)$ has support $I^{c}$, or $\sigma G(a)$ contains two hoopings with support $I^{c}$ and opposite signs.

ii) There exists $a \in \partial \Omega=\Omega-\stackrel{\circ}{\Omega}, I \subset I(a)$ and hoopings $C_{I}$ and $C_{i}$, for each $i \in I$, in $\sigma G(a)$ such that $\#(I) \geq 2$, the support of $C_{I}$ (resp. $C_{i}$ ) is $I^{c}$ (resp. $\left.\{i\}^{c}\right)$, and

$$
\operatorname{sgn}\left(C_{I}\right) \prod_{i \in I} \operatorname{sgn}\left(C_{i}\right)>0 .
$$

Furthermore, either $\#(I)$ is even or there exist $b \in \partial \Omega, I^{\prime} \subset I(b), C_{I^{\prime}}, C_{j}$, $j \in I^{\prime}$, with similar properties as above and

$$
\operatorname{sgn}\left(C_{I^{\prime}}\right) \prod_{j \in I^{\prime}} \operatorname{sgn}\left(C_{j}\right)<0 .
$$

Proof. Again, it is enough to treat the case $\sigma=1$. When $F$ is not univalent we know from Theorem 7' that there is $a \in \Omega$ and $I \subset I(a)$ such that

$$
m_{I}(a) \prod_{i \in I} m_{i}(a) \leq 0 \text {. }
$$

Assume $m_{I}(a)=0$. Then, by Lemma 2 ii), the statement i) must hold. Assume now, that, for all $J \subset I(a), m_{J}(a) \neq 0$. Since

$$
m_{I}(a) \prod_{i \in I} m_{i}(a)<0
$$


we must have $\#(I) \geq 2$. Furthermore, for every vertex $k \in V$, if we replace $F$ by $\tau_{k} F=\tau_{\{k\}} F$ the quantity $m_{I}(a)$ is multiplied by +1 (resp. -1 ) if $k \in I$ (resp. $\left.k \in I^{c}\right)$. Therefore, in all cases, $m_{I}(a) \prod_{i \in I} m_{i}(a)$ gets multiplied by $(-1)^{\#(I)}$. It is thus invariant if and only if \#(I) is even. If this number is odd, we apply the same discussion to $-F$ and we get $b$ and $I^{\prime}$ such that

$$
m_{I^{\prime}}(b) \prod_{j \in I^{\prime}} m_{j}(b)>0 .
$$

Using (8), the statement i) follows.

\section{3}

Let $\Omega=\prod_{i=1}^{n} \Omega_{i}$ be an arbitrary product of intervals as in 2.1 and let $F: \Omega \rightarrow \mathbb{R}^{n}$ be a $C^{1}$ map. From Theorem 7 one gets some information on where two zeroes of $F$ can be:

Theorem 8. Fix $\sigma \in \Sigma_{n}$. Assume that there exist two points a and $b$ in $\Omega$ such that $F(a)=F(b)$. Assume furthermore that, when $x$ lies in $\stackrel{\circ}{\Omega}$, all hoopings of $\sigma G(x)$ are negative. Then there exists $x \in \Omega$ such that, when $x_{i} \in \partial \Omega_{i}$, the $i$-th coordinate of $a$ or $b$ is equal to $x_{i}$, and a subset $I \subset I(x)$ such that no hooping in $\sigma G(x)$ has support equal to $I^{c}$.

Proof. One can find a bounded closed product of intervals $\Omega^{\prime} \subset \Omega$ containing $a$ and $b$ and such that, whenever $x \in \Omega^{\prime}$ and $x_{i} \in \partial \Omega_{i}$, the $i$-th coordinate of $a$ or $b$ is equal to $x_{i}$. Since the restriction of $F$ to $\Omega^{\prime}$ is not univalent, we can apply Theorem 7 . We are not in case ii) because, $J(x)$ being continuous in $x$, for every $x \in \Omega^{\prime}$ all the hoopings of $G(x)$ are nonpositive.

Therefore Theorem $7 \mathrm{i}$ ) holds true for some $x \in \Omega^{\prime}$, hence the conclusion.

q.e.d.

\section{The algebraic case}

Assume now that $\Omega=\mathbb{R}^{n}$ and that $F$ is $C^{1}$ and algebraic, by which we mean that its graph $\{(x, F(x))\} \subset \mathbb{R}^{n} \times \mathbb{R}^{n}$ is the set of zeroes of a family of real polynomials in $2 n$ variables. This will be the case for instance when each component $f_{i}$ of $F$ is the quotient of two polynomials in $n$ variables. In that case, a result of Campbell [2] leads to a stronger conclusion than Theorem 5 1).

Theorem 9. Choose any ordering of $V$. Let

$$
F: \mathbb{R}^{n} \rightarrow \mathbb{R}^{n}
$$


be a $C^{1}$ map which is not univalent. Then we can choose $k \leq n$ such that, if I consists of the first $k$ vertices,

i) Either $G(F)$ has two hoopings of opposite signs and support equal to I;

ii) Or there exists $a \in \mathbb{R}^{n}$ such that none of the hoopings of $G(a)$ has support equal to $I$.

Proof. For any $k \leq n$ and $a \in \mathbb{R}^{n}$ we let

$$
d_{k}(a)=\operatorname{det}\left(J(a)_{I}\right) .
$$

According to [2], if $d_{k}(a)>0$ for all $k=1, \ldots, n$, the map $F$ is univalent. Therefore, under our assumption, $d_{k}(a) \leq 0$ for some $a$ and some $k$. When $d_{k}(a)=0$, either i) or ii) is true (by Lemma 2 ii)). Given $i \in V$, when we replace $F$ by $\tau_{i} F, d_{k}(a)$ gets multiplied by -1 (resp. +1$)$ if $i \leq k$ (resp. $i>k$ ). So we can assume that, for every $I \subset V$, there exist $k$ and $a$ such that $\tau_{I} d_{k}(a)<0$ (with obvious notation). By replacing $F$ by $\tau_{1} F$ we see that we can also assume that there exist $k^{\prime}$ and $b$ such that $\tau_{I} d_{k^{\prime}}(b)>0$.

Unless i) or ii) holds, we can assume (using Lemma 2 again) that for every $I \subset V$ and every $k \leq n$ the sign of $\tau_{I} d_{k}(x)$ does not depend on $x \in \mathbb{R}^{n}$. Let then $m$ be the maximum of all integers $p$ such that, if $i \leq k \leq p, d_{k}(x)$ and $d_{1}(x)$ have the same sign. When $m<n$, we can increase $m$ by $m+1$ by replacing $F$ by $\tau_{m+1} F$. By repeating this process, we find $I$ such that $\tau_{I} d_{k}(x)$ has a fixed sign for every $k \leq n$ and every $x \in \mathbb{R}^{n}$. As we saw in the previous paragraph, this cannot happen.

q.e.d.

\section{References}

[1] P. Alexandroff, H. Hopf, Topologie, Band 1, Berichtigter Reprint, Die Grundlehren der mathematischen Wissenschaften, Band 45, BerlinHeidelberg-New York, Springer-Verlag, XIII, (1974), 636 p.

[2] L.A. Campbell, Rational Samuelson maps are univalent, J. Pure Appl. Algebra 92, No.3 (1994), 227-240.

[3] O. Cinquin, J. Demongeot, Positive and negative feedback: striking a balance between necessary antagonists, J. Theoretical Biology 216 (2002), 229241.

[4] J.Eisenfeld, C. DeLisi, On conditions for qualitative instability of regulatory circuits with application to immunological control loops, in Mathematics and Computers in Biomedical Applications, J.Eisenfeld and C.DeLisi eds., Elsevier, Amsterdam (1985), 39-53. 
[5] D. Gale, H. Nikaido, The Jacobian matrix and global univalence of mappings, Math. Annalen 159 (1965), 81-93.

[6] C.B. Garcia, W.I. Zangwill, On univalence and P-matrices, Linear Algebra Appl. 24, (1979), 239-250.

[7] J.-L. Gouzé, Positive and negative circuits in dynamical systems, J. Biol. Sys. 6 (1998), 11-15.

[8] M.S. Gowda, G. Ravindran, Algebraic univalence theorems for nonsmooth functions, J. Math. Anal. Appl. 252, No.2, (2000), 917-935.

[9] M. Kaufman, R. Thomas, Model analysis of the the bases of Multistationarity in the Humoral Immune Response, J. Theoretical Biology 129 (1987), 141-162.

[10] T. Parthasarathy, On global univalence theorems, Lecture Notes in Mathematics, 977 (1983), Berlin-Heidelberg-New York, Springer-Verlag .

[11] E. Plahte, T. Mestl, W.S. Omholt, Feedback circuits, stability and multistationarity in dynamical systems, J. Biol. Sys. 3 (1995), 409-413.

[12] E.H. Snoussi, Necessary conditions for multistationarity and stable periodicity, J. Biol. Sys. 6 (1998), 3-9.

[13] R. Thomas, On the relation between the logical structure of systems and their ability to generate multiple steady states or sustained oscillations, Springer Series in Synergetics, 9 (1981), 180-193.

[14] R. Thomas, Logical description, analysis, and feedback loops, in Aspects of Chemical Evolution, G. Nicolis ed., XVII-th Solvay Conference on Chemistry (1980), John Wiley and Sons, 247-282.

[15] R. Thomas, M. Kaufman, Multistationarity, the basis of cell differentiation and memory. I. Structural conditions of multistationarity and other nontrivial behaviour, Chaos 11 (2001), 170-179.

Keywords : interaction graph, multistationarity, Jacobian matrix, global univalence. 